\title{
The effect of subminimal inhibitory concentrations of antibiotics on the adherence ability of Pseudomonas aeruginosa to epithelial cells in vitro
}

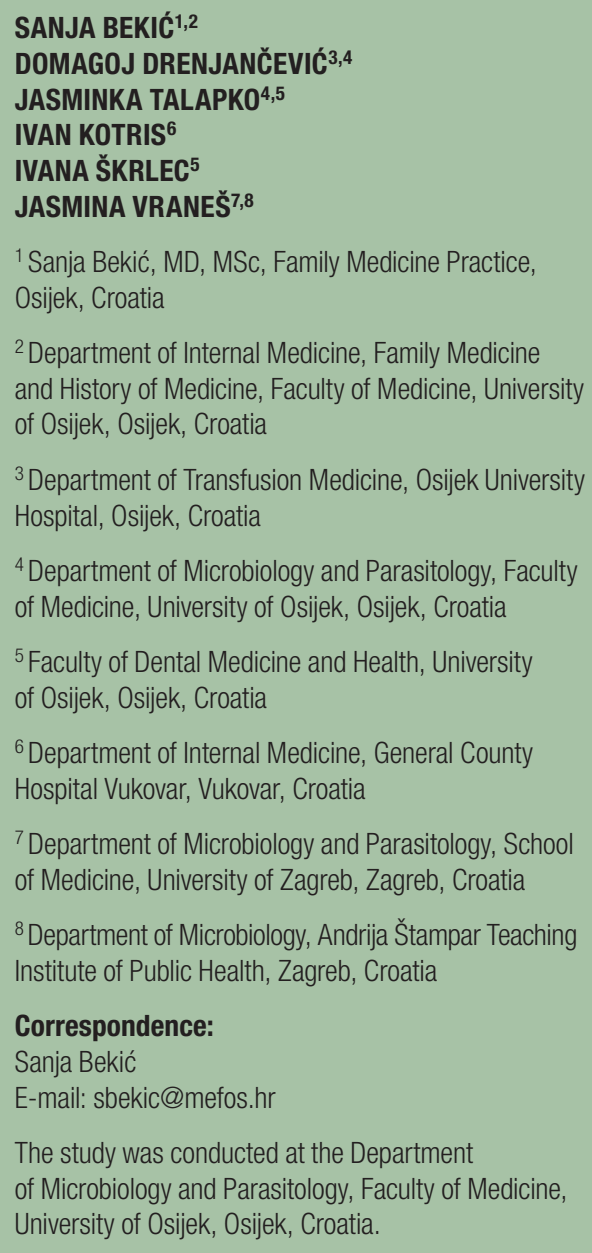

${ }^{3}$ Department of Transfusion Medicine, Osijek University Hospital, Osijek, Croatia

${ }^{4}$ Department of Microbiology and Parasitology, Faculty of Medicine, University of Osijek, Osijek, Croatia

${ }^{5}$ Faculty of Dental Medicine and Health, University of Osijek, Osijek, Croatia

${ }^{6}$ Department of Internal Medicine, General County Hospital Vukovar, Vukovar, Croatia

${ }^{7}$ Department of Microbiology and Parasitology, School of Medicine, University of Zagreb, Zagreb, Croatia

${ }^{8}$ Department of Microbiology, Andrija Štampar Teaching Institute of Public Health, Zagreb, Croatia

\section{Correspondence:}

Sanja Bekić

E-mail: sbekic@mefos.hr

The study was conducted at the Department of Microbiology and Parasitology, Faculty of Medicine, University of Osijek, Osijek, Croatia.

Keywords: Ceftazidime; Ciprofloxacin; Gentamicin; Bacterial adherence

Source of support

The authors of this publication declare that this study was supported by grant No. 0219281 from the Ministry of Science, Education, and Sport, Republic of Croatia.

\begin{abstract}
Background and purpose: The aim of this study was to examine the influence of subminimal inhibitory concentrations (subMICs) of ceftazidime, ciprofloxacin and gentamicin on the adherence ability and morphology of wild-type Pseudomonas aeruginosa strains to the Buffalo green monkey kidney cell line, using indirect immunofluorescence staining.
\end{abstract}

Materials and methods: Bacterial adherence changes were tested before and after exposure to 1/2,1/4, 1/8,1/16 and 1/32 MIC of antibiotics.

Results: A statistical difference in the number of attached bacteria after exposure to all subMICs of ceftazidime and ciprofloxacin was observed $(p<0.05)$, even after only 1/2 MIC of gentamicin.

Conclusion: The results of this study have shown that antibiotics in much lower concentrations than those necessary for inhibition of bacterial multiplications could damage the adherence of Pseudomonas aeruginosa to the epithelial cell line.

\section{INTRODUCTION}

$\mathbf{B}$ acterial adherence is the first step in the pathogenesis of most bacte-

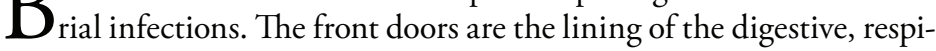
ratory and urinary tracts, the skin and conjunctiva. The process of adherence is mediated by molecules of adhesion and by complementary receptors on the cell surface. The interaction is stereospecific, and the most common bacterial adhesins, which cause infections in human, are proteins, lipopolysaccharides, and lipoprotein molecule, which are parts of the bacterial cell wall (1).

Pseudomonas aeruginosa has multiple mechanisms to adhere to host cells. The most important adhesins are fimbriae, alginate and exoenzyme

\footnotetext{
Nonstandard abbreviations

BGMK - Buffalo green monkey kidney cell line

ITFA - indirect immunofluorescence staining

MIC - minimal inhibitory concentrations

PBS - phosphate buffered saline

subMIC - subminimal inhibitory concentrations
}

Received April 10, 2018.

Revised July 02, 2018.

Accepted July 24, 2018. 
$S(2,3)$. The strains with mutations in their genes for fimbriae function, or without fimbriae, show reduced adherence ability $(4,5)$. Not only are fimbriae an important virulence factor, but they are also important for their ability to move across a smooth surface with "twitching motility", extension and retraction of the pili, which is particularly important for the formation of microcolonies and biofilm. P. aeruginosa infections are complicated to treat because the bacteria have a substantial intrinsic resistance to certain antibiotics. One of the possibilities for preventing bacterial infection lies in action directed at the active substance in the process of bacterial adherence. The antibiotic concentration in the serum does not always reflect the concentration at the cellular level. In the infected area, antibiotics are often present at subminimal inhibitory concentrations (subMICs). Antibiotic subMICs can inhibit the formation and expression of adhesins. It was previously described that subMICs antibiotics could prevent the propagation of bacteria in vitro (6). Also, it can change the expression, number, morphology, and function of fimbriae $(7,8)$. New findings on bacterial adherence and its relationship with antibiotics could provide us with a better insight into the prevention and treatment of infection in the initial phase. The aim of this study was to examine the influence of subMICs $(1 / 2,1 / 4$, $1 / 8,1 / 16$ and 1/32 MIC) of ceftazidime, ciprofloxacin and gentamicin on the adherence ability of wild-type Pseudomonas aeruginosa strains, isolated from various clinical sites to the Buffalo green monkey kidney (BGMK) cell line, using indirect immunofluorescence staining (ITFA).

\section{MATERIALS AND METHODS}

\section{Bacterial strains}

The study included 30 strains of $P$. aeruginos $a$ obtained from various clinical specimens during routine diagnostics. After biochemical identification, the strains were stored in a deep reserve agar and subcultured at the Department of Microbiology and Parasitology, Faculty of Medicine, University of Osijek, Croatia, where the research was conducted. The study was approved by the Ethics Committee of the Faculty of Medicine, the Josip Juraj Strossmayer University of Osijek and by the Ethics Committee of the University Hospital Osijek, Croatia.

\section{Adherence of P. aeruginosa strains to epithelioid cell line testing}

Standard continuous epithelioid cell culture BGMK was used for adherence testing. The BGMK cell culture was developed on coverslips, sized $11 \times 22 \mathrm{~mm}$. The coverslips with the cells were immersed in tubes containing suspensions of the tested strains, which were adjusted to an optical density of 1MF (McFarland, API SYSTEM). After incubation for one hour at $37^{\circ} \mathrm{C}$ using a shaker $(50$ rpm), the cell culture was washed with PBS (phosphate buffered saline) and fixed with acetone. After fixation, hyperimmune serum containing antibodies against the bacterial O-antigen (Diagnostic serums, Institute of Immunology, Zagreb) was instilled, followed by incubation in a humid chamber for 30 minutes, at a temperature of $37^{\circ} \mathrm{C}$. The coverslip cell cultures were washed, after which secondary antibodies were instilled, labelled with fluorescein isothiocyanate (Diagnostic sera, Institute of Immunology, Zagreb) and incubated in a humid chamber for 30 minutes at a temperature of $37^{\circ} \mathrm{C}$. After washing the cell culture, the preparation was dyed with Evans methylene blue and observed under a fluorescence microscope (Zeiss Axiovert 25). For each strain preparation, 40 cells were microscoped and the average number of bacteria that had adhered per cell, in the cell culture, was determined. All testing was done in triplicate, before and after exposure to the subMICs of the selected antibiotics.

\section{Antimicrobial agent susceptibility testing}

Susceptibility testing to ceftazidime, gentamicin, and ciprofloxacin was performed by the standard broth dilution method. For each examined strain of $P$. aeruginosa, the minimum inhibitory concentration (MIC) was determined (9).

These three antibiotics are selected because they have different mechanisms of action. Ceftazidime belongs to beta-lactam antibiotics that interfere with the synthesis of peptidoglycan and thus directly affect the bacteria cell wall. Ciprofloxacin belongs to a group of quinolones which inhibit the enzyme bacterial DNA gyrase and thus inhibit the replication of bacterial DNA. Gentamicin is an aminoglycoside antibiotic that interacts with the $16 \mathrm{~S}$ rRNA of the $30 \mathrm{~S}$ ribosome subunit, and cause the misreading and premature termination of translation of bacterial mRNA (10).

\section{SubMICs of antibiotics}

The bacteria' strains were exposed to $1 / 2,1 / 4,1 / 8$, 1/16, 1/32 MIC of ceftazidime, gentamicin, and ciprofloxacin. Part of a single colony of each strain was inoculated in three millilitres of nutrient broth, previously divided into test tubes $\left(-10^{6}\right.$ bacteria $\left./ \mathrm{ml}\right)$. The tubes were incubated at $37^{\circ} \mathrm{C}$ for two hours, and during this time the studied strain reached the logarithmic phase of multiplication. The bacterial culture broth was mixed with a suspension of antibiotics in a series of tubes in a ratio of 1:1 to final concentrations of $1 / 2,1 / 4,1 / 8,1 / 16,1 / 32$ MIC made for each antibiotic. For each antibiotic, two test tubes were used to determine the minimum inhibition concentration for each strain, and twice the MIC (2 MIC). Dilutions of antibiotics greater than the subinhibitory concentration (1 MIC and 2 MIC) were used as the control for the regularity of the procedure. Further incubation was continued for four hours. After a total of 
Table 1. Number of adherent bacteria to BGMK cell line before and after exposure of subMICs of ceftazidime, ciprofloxacin, and gentamicin

\begin{tabular}{|c|c|c|c|}
\hline & CEFTAZIDIME & CIPROFLOXACIN & GENTAMICIN \\
\hline WITHOUT ATB & & $59.52 \pm 22.45$ & \\
\hline 1/2 MIC & $3.03 \pm 2.39^{*}$ & $3.4 \pm 3.64^{*}$ & $56 \pm 20.84^{*}$ \\
\hline 1/4 MIC & $6.93 \pm 3.11^{*}$ & $10.07 \pm 5.74^{*}$ & $57.70 \pm 20.16$ \\
\hline 1/8 MIC & $12.4 \pm 5.14^{*}$ & $15.27 \pm 6.8^{*}$ & $58.77 \pm 21.52$ \\
\hline 1/16 MIC & $18.23 \pm 7.82^{*}$ & $36.67 \pm 24.22^{*}$ & $61.9 \pm 22.83$ \\
\hline 1/32 MIC & $35.03 \pm 15.39^{*}$ & $54.97 \pm 23.95^{*}$ & $63.2 \pm 23.38$ \\
\hline
\end{tabular}

$\mathrm{N}=30$, data represent as mean \pm standard deviation, ATB - antibiotic, ${ }^{*} \mathrm{p}<0.05$, Friedman test

Table 2. Differences between subMICs of ceftazidime, ciprofloxacin, and gentamicin on the adherence ability of P. aeruginosa strains in epithelioid cell culture

\begin{tabular}{|c|c|c|c|c|c|}
\hline $\mathrm{N}=30$ & $1 / 2$ & $1 / 4$ & $1 / 8$ & $1 / 16$ & $1 / 32$ \\
\hline \multirow{2}{*}{ CEFTAZIDIME - CIPROFLOXACIN } & $Z=0.129$ & $Z=2.555$ & $Z=2.173$ & $Z=3.733$ & $Z=3.429$ \\
\hline & $\mathrm{p}=0.898$ & $\mathrm{p}=0.011$ & $\mathrm{p}=0.030$ & $\mathrm{p}<0.001$ & $\mathrm{p}=0.001$ \\
\hline \multirow{2}{*}{ CEFTAZIDIME - GENTAMICIN } & $Z=4.782$ & $Z=4.782$ & $Z=4.782$ & $Z=4.782$ & $Z=4.541$ \\
\hline & $\mathrm{p}<0.001$ & $\mathrm{p}<0.001$ & $\mathrm{p}<0.001$ & $\mathrm{p}<0.001$ & $\mathrm{p}<0.001$ \\
\hline \multirow{2}{*}{ CIPROFLOXACIN - GENTAMICIN } & $Z=4.782$ & $Z=4.782$ & $Z=4.782$ & $Z=4.566$ & $Z=3.581$ \\
\hline & $\mathrm{p}<0.001$ & $\mathrm{p}<0.001$ & $\mathrm{p}<0.001$ & $\mathrm{p}<0.001$ & $\mathrm{p}<0.001$ \\
\hline
\end{tabular}

Data were compared using Wilcoxon test

six hours of incubation at $37^{\circ} \mathrm{C}$, each tube was centrifuged, the broth was decanted, and subsequently, the strain was washed three times (PBS, pH 7.2, 300 $\mathrm{mOsmol} / \mathrm{l})$ and adjusted to an optical density of $1 \mathrm{McFar}-$ land (API System).

\section{Statistical analysis}

The normal distribution was checked using the Kolmogorov-Smirnov test with Lilliefors correction empirical significance level (p-value), and the Shapiro-Wilks. The significance of differences in the activity of all five subMICs of the antibiotics ceftazidime, ciprofloxacin and gentamicin on the ability for bacterial adherence was tested by the Friedman test and the Wilcoxon signed-rank test. Statistically significant differences were confirmed at the level of $\mathrm{p}<0.05$. Preparation of data and the statistical analysis were performed using a Microsoft Excel 2003 computer spreadsheet. In the analysis, we used the statistical software package SPSS 15.0 for Windows, Evaluation Version and Statistica 6.0.

\section{RESULTS}

The number of adherent bacteria, before and after exposure to the antibiotics, was determined by the average triplicate scores (arithmetic mean \pm standard deviation). Most $P$. aeruginosa strains adhered very well to the epi- thelioid cell culture used: the number of adherent bacteria per cell ranged between 30 and 105, with a median of 54 bacteria (interquartile range: 40.33-80.00). The overall effect of all three tested antibiotics is shown in Table 1.

\section{The ability of bacterial adherence after exposure to ceftazidime subMICs}

The ability of bacterial adherence after exposure to ceftazidime subMICs is shown in Table 1. It may be concluded that with decreasing concentrations of ceftazidime there is an increase in the number of adherent bacteria. The average number of bacteria at 1/32 MIC was 11.549 times higher than at 1/2 MIC. The median shows a similar tendency. With decreasing concentrations of antibiotics, the range of variation increased. An increase may be observed in median values and the interquartile range with decreasing concentrations of ceftazidime. Some extreme values exist in distributions relating to the 1/8,1/16 and 1/32 MIC of ceftazidime. There were no extreme values in any distribution, while outliers exist in distributions relating to the $1 / 8,1 / 16$ and $1 / 32 \mathrm{MIC}$ of ceftazidime. Ceftazidime is effective in preventing bacterial adherence only at the concentration of $1 / 2$ MIC. All the differences in the activity of the subinhibitory concentrations of ceftazidime on the ability for bacterial adherence were statistically significant at the significance level of $\mathrm{p}<0.05$. 


\section{The ability of bacterial adherence after exposure to ciprofloxacin subMICs}

The number of bacteria which adhered to the continuous epithelioid cell culture before and after exposure to ciprofloxacin subMICs is shown in Table 1. The average number of adherent bacteria at a minimum concentration of antibiotic ciprofloxacin (1/32 MIC) was 16.17 times higher than at the maximum concentration (1/2 MIC). At the lowest concentration of the antibiotic (1/32 MIC), the average number of adhered bacteria approached the average values recorded as the initial values. The median value at a concentration of antibiotics of 1/32 MIC was 25 times higher than in the case of concentrations of $1 / 2$ MIC. With a lower concentration of the antibiotic ciprofloxacin in the test system, there is an increase in the difference between the median and the interquartile range. In the case of the antibiotic analysed, extreme values were observed in the distributions relating to the ability of bacterial adherence in the two highest concentrations of the antibiotic. The efficiency of ciprofloxacin is decreased with dilution, so at $1 / 32$ MIC the number of adhered bacteria was approximately the same as that without antibiotic. According to the Wilcoxon test, all the differences in the activity of the subMICs of the antibiotic ciprofloxacin on the ability of bacterial adherence were statistically significant $(\mathrm{p}<0.05)$.

\section{The ability of bacterial adherence after exposure to gentamicin subMICs}

The number of bacteria which adhered to the BGMK cell culture after exposure to gentamicin subMICs is shown in Table 1 . The average numbers of adherent bacteria after exposure to all the subMICs of gentamicin are very similar to the initial values. Only in the case of $1 / 2$ MIC of gentamicin, there were statistically significant fewer adherent bacteria on the cell line than before exposure to the antibiotic (Wilcoxon test, $\mathrm{p}<0.05$ ). From these results, we may conclude that gentamicin has a less significant effect on reducing the ability of bacterial adherence than ceftazidime and ciprofloxacin.

\section{Differences in the action of the antibiotics}

The statistically significant differences between ceftazidime, ciprofloxacin, and gentamicin on the adherence ability of strains of $P$. aeruginos $a$ in continuous epithelioid cell culture are shown in Table 2. For all subMICs, ceftazidime and ciprofloxacin were significantly more effective in reducing the ability of bacterial adherence compared to gentamicin. According to the Wilcoxon test, at the level of $p<0.001$, there was a statistically significant difference in the reduction of the ability for bacterial adherence between the antibiotic gentamicin and the other two antibiotics. However, there were significant differences between the effects of the antibiotics ceftazidime and ciprofloxacin, except at 1/2 MIC. As shown in Table 2 , ceftazidime had a statistically significantly stronger effect compared to the similar subMIC of ciprofloxacin. It may be concluded, according to the results presented, that the subMICs of ceftazidime had the most prominent effect on inhibiting bacterial adherence ability and gentamicin had the least effect.

\section{DISCUSSION}

Subinhibitory concentrations of antibiotics may affect various bacterial virulence factors. They can act on the morphology and the antigenicity of the bacteria $(11,12)$, on the growth of bacteria (13), on the synthesis and secretion of enzymes $(14,15)$ and can act on bacterial adherence $(16,17)$. All these factors contribute to the reduction of bacterial pathogenicity. Bacterial exposure to the subMIC of antibiotics inhibited the ability of adherence, which is an important virulence factor $(18,19)$.

This research studies the effect of subMIC ceftazidime, ciprofloxacin, and gentamicin on the adherence ability of $P$. aeruginosa on the epithelioid cells of BGMK cell culture, in five different concentrations of antibiotics, 1/2, 1/4, 1/8, $1 / 16$, and 1/32 MIC. Certain antibiotics deplete the ability for bacterial adherence, which depends on the type of antibiotic, antibiotic concentration in the test system and the characteristics of the strain $(20,21)$. The anti-adhesive effect of antibiotics is manifested in the subsequent inactivation of synthesis or the expression of adhesins on the bacterial surface, which leads to the formation of less valuable functional adhesin, or the release of adhesins from the bacterial surface. It could also lead to a morphological change in the bacteria, such as forming filaments, resulting in no stereospecific reactions of bacterial adhesins with the receptor of the host cell occur (22), and the bacteria become more susceptible to phagocytosis (23).

In our study, ceftazidime, and ciprofloxacin significantly reduced adherence ability. With decreasing concentrations of antibiotics, there was an increase in the number of adherent bacteria in the analyzed strains. At the concentration of 1/32 MIC for ciprofloxacin, and at concentrations of $1 / 16$ and $1 / 32 \mathrm{MIC}$ for gentamicin, the number of adherent bacteria was approaching the number of bacteria at the initial value. The antibiotic gentamicin showed a significantly lower impact in the examined concentrations on the reduction of adherence of $P$. aeruginos $a$, compared to ceftazidime and ciprofloxacin. The inefficiency of gentamicin with a statistically significant difference of $\mathrm{p}$ $<0.05$, appeared at all concentrations, except 1/2 MIC. The number of adherence bacteria in those concentrations was similar to the number of bacteria at the initial values.

The aim of the study was to examine the differences in the operation of three analyzed antibiotics on the adherence ability of strains of Pseudomonas aeruginosa on continuous epithelioid cell cultures. According to the selected statistical indicators, the highest average rank was 
determined by the variable that represents the ability of bacterial adherence in the use of the antibiotic gentamicin and for all the subinhibitory concentrations. On this basis, it was established that gentamicin, at all tested concentrations, affects the ability of bacterial adherence the least, compared to ceftazidime and ciprofloxacin, while ciprofloxacin proved to be more effective than gentamicin, and ceftazidime the most effective in all tested concentrations. At a concentration of 1/2 MIC, ceftazidime and ciprofloxacin were significantly more effective in reducing the ability of bacterial adherence, compared to gentamicin. There was no confirmed statistically significant difference between the efficacy of ceftazidime and ciprofloxacin at $1 / 2 \mathrm{MIC}$, at the level of statistical significance of $\mathrm{p}<0.05$ (Table 2).

The results of this study support the hypothesis that bacterial adhesin protein mediates the adhesion of $P$. aeruginosa to BGMK epitheloid cell culture and that during synthesis these membrane proteins may be inhibited by lower concentrations of antibiotics. P. aeruginosa protein structure is classified as $\mathrm{N}$-methylphenylalanine fimbriae. Earlier studies have shown that subMICs of azithromycin inhibit the expression of fimbriae Neisseria gonorrhoeae, which has the same $\mathrm{N}$-methylphenylalanine structure fimbriae as $P$. aeruginosa $(24,25)$. A series of studies demonstrated that subinhibitory concentrations of antibiotics reduce the ability of bacteria adherence. Fonesca et al. (26) described the reduced ability of adherence of $P$. aeruginosa after exposure to subMICs of piperacillin and tazobactam. Vidaya et al. observed that subMIC of different antibiotics reduced the adhesive ability of $E$. coli to uroepithelial cells (27) and that co-trimoxazole was the most effective. Sandberg et al. (22) observed that elongated, socalled filamentous forms of bacteria, formed after exposure to subMIC beta-lactam antibiotics, had a lower ability to adhere to the receptors of uroepithelial cells. The observed anti-adhesive effect of antibiotics not only tells us about the biophysicochemical changes in bacterial cells but also gives us information about the actual events in the body caused by the action of low-dose antibiotics. With the intermittent application of antibiotics, which is common in the treatment of bacterial infections, antibiotic concentrations in the blood, as well as at the site of the infection, will depend upon the dose of antibiotic, the mode of administration, and the patient's immune system. At the site of infection, the concentration of antibiotics administered may exceed the MIC for a particular time, but eventually, reach the value of MIC and finally fall to a subMIC value, usually between doses (28). In the case of tissue infection, such as an abscess or chronic osteomyelitis, the concentration of antibiotics in the tissues is often lower than in the blood (28). By inhibiting adherence with antibiotic subMICs, an emerging infection is prevented in the first step. In vivo research of antibiotic subMICs is rare because it is difficult to assess the results objectively. Many research are done in vitro, because of controlled condi- tions. The intention is to transfer events in vivo using specific experimental systems. Accordingly, as observed in this experimental study, antibiotics in their subminimal inhibitory concentrations have anti-adhesive effects and as a result could play a significant role in reducing the pathogenicity of $P$. aeruginos $a$, thus preventing and controlling infections caused by these microorganisms.

\section{CONCLUSIONS}

Although research about the possibilities of preventing bacterial adherence with subminimal inhibitory concentrations of antibiotics is frequent (18), the results are sometimes confusing and often it is hard to compare results and draw general conclusions. The differences are numerous: researchers investigate the effect of subMIC of different bacterial species, with various strains within the same species, or recombinant strains, the bacterial suspensions are in contact with the antibiotics for different lengths of time and are added at different stages of multiplication of the bacterial population. In experimental systems, intentionally or spontaneously exfoliated epithelial cells of different tissues or cell cultures are used, and the bacterial adherence capability is different. The cell culture of green monkey kidney is an excellent tool for further research, which, together with future research into the activities of subMICs of antibiotics on the ability of bacterial adherence, will provide additional information about the activity and the action of subMICs of antibiotics on bacterial cells. This research will allow the application of subMIC of antibiotics in the prevention and treatment of infections.

Of the three tested antibiotics, the most effective proved to be ceftazidime, which significantly reduced the ability of bacterial adherence in all five subMICs. Ciprofloxacin had the same effect, but less ability to reduce bacterial adherence compared to ceftazidime. At the concentration of $1 / 2$ MIC, there was no statistically significant difference between the activity of ceftazidime and ciprofloxacin, while in all other tested concentrations of the two antibiotics the differences in the effect were significant. A statistically significant difference was determined between the activity of ceftazidime and gentamicin in all tested concentrations, as well as between ciprofloxacin and gentamicin. Gentamicin only affected bacterial adherence at concentrations of 1/2 MIC.

By comparing the efficacy of all three tested antibiotics at all of the mentioned concentrations, ceftazidime proved to be the most effective while gentamicin had the least effect on reducing bacterial adherence. Ciprofloxacin is more effective than gentamicin but less efficient than ceftazidime. The results of this study showed that antibiotics could damage the bacterial ability to adhere to the cell surface at the concentration much lower than those necessary to inhibit bacterial multiplication (MIC). Prevention of bacterial adherence is an attractive way of preventing and protecting against infectious diseases. 


\section{REFERENCES}

1. BERNE C, DUCRET A, HARDY GG, BRUN YV 2015 Adhesins involved in attachment to abiotic surfaces by Gram-negative bacteria. Microbiol spectr 3(4).

https://doi.org/10.1128/microbiolspec.MB-0018-2015

2. LAVERTY G, GORMAN SP, GILMORE BF 2014 Biomolecular Mechanisms of Pseudomonas aeruginosa and Escherichia coli Biofilm Formation. Pathogens 3(3): 596-632.

https://doi.org/10.3390/pathogens3030596

3. RANGEL SM, DIAZ MH, KNOTEN CA, ZHANG A, HAUSER AR 2015 The Role of ExoS in Dissemination of Pseudomonas aeruginosa during Pneumonia. PLoS Pathog 11(6):e1004945. https://doi.org/10.1371/journal.ppat.1004945

4. HAHN HP 1997 The type-4 pilus is the major virulence-associated adhesin of Pseudomonas aeruginosa - a review. Gene 192(1): 99-108. https://doi.org/10.1016/S0378-1119(97)00116-9

5. WOZNIAK DJ, KEYSER R 2004 Effects of subinhibitory concentrations of macrolide antibiotics on Pseudomonas aeruginosa. Chest 125(Suppl 2): 62S-69S. https://doi.org/10.1378/chest.125.2_suppl.62S

6. SCHIERHOLZ JM, BEUTH J, PULVERER G 1999 Adherent bacteria and activity of antibiotics. J Antimicrob Chemother 43(1): 158-60. https://doi.org/10.1093/jac/43.1.158

7. GONEAU LW, HANNAN TJ, MACPHEE RA, SCHWARTZ DJ, MACKLAIM JM, GLOOR GB, RAZVI H, REID G, HULTGREN SJ, BURTON JP 2015 Subinhibitory antibiotic therapy alters recurrent urinary tract infection pathogenesis through modulation of bacterial virulence and host immunity. mBio 6(2):e00356-15. https://doi.org/10.1128/mBio.00356-15

8. BECEIRO A, TOMÁS M, BOU G 2013 Antimicrobial Resistance and Virulence: a Successful or Deleterious Association in the Bacterial World? Clin Microbiol Rev 26(2): 185-230. https://doi.org/10.1128/CMR.00059-12

9. CLSI. 2017 Performance Standards for Antimicrobial Susceptibility Testing. 27th ed. CLSI supplement M100. Wayne, PA: Clinical and Laboratory Standards Institute

10. KAPOOR G, SAIGAL S, ELONGAVAN A 2017 Action and resistance mechanisms of antibiotics: A guide for clinicians. J Anaesthesiol Clin Pharmacol 33: 300-5. https://doi.org/10.4103/joacp.JOACP_349_15

11. HOŠTACKA A, ČIŽNAR I, SLOBODNIKOV AL, KOTULOVA D 2006 Clinical Pseudomonas aeruginosa: potential factors of patogenicity and resistance of antimicrobials. Folia Microbiol (Praha) 51(1): 633-8. https://doi.org/10.1007/BF02931631

12. ISHIKAWA J, HORII T 2005 Effects of mupirocin at subinhibitory concentrations on biofilm formation in Pseudomonas aeruginosa. Chemotherapy 51: 361-362. https://doi.org/10.1159/000088962

13. LORIAN V 1993 Medical relevance of low concentrations of antibiotics. J Antimicrob Chemother 31(Suppl D): 137-48. https://doi.org/10.1093/jac/31.suppl_D.137

14. BRAGA PC, SASSO MD, SALA MT 2000 Sub-MIC concentrations of cefodizime interfere with various factors affecting bacterial virulence. J Antimicrob Chemother 45(1): 15-25. https://doi.org/10.1093/jac/45.1.15

15. SONSTEIN SA, BERNHAM J 1993 Effects of low concentrations of quinolone antibiotics on bacterial virulence mechanisms. Diagn
Microb Infect Dis 16(4): 277-89.

https://doi.org/10.1016/0732-8893(93)90078-L

16. WILSON JW, SCHURR MJ, LEBLANC CL, RAMAMURTHYR, BUCHANAN KL, NICKERSON CA 2000 Mechanisms of bacterial pathogenicity. Postgrad Med J 78(918): 216-24. https://doi.org/10.1136/pmj.78.918.216

17. DWIDAR M, LEUNG BM, YAGUCHI T, TAKAYAMA $S$, MITCHELL RJ 2013 Patterning Bacterial Communities on Epithelial Cells. PLoS ONE 8(6):e67165. https://doi.org/10.1371/journal.pone.0067165

18. KAWAMURA-SATO K, IINUMA Y, HASAGAWA T, HORRI T, YAMASHITO T, OHTA M 2000 Effect of subinhibitory concentrations of macrolides on expression of flagellin in Pseudomonas aeruginosa and Proteus mirabilis. Antimicrob Agents Chemother 44(Suppl 10): 2869-72.

https://doi.org/10.1128/AAC.44.10.2869-2872.2000

19. SCHIFFERLI DM, BEACHEY EH 1988 Bacterial adhesion: modulation by antibiotics with primary targets other than protein synthesis. Antimicrob Agents Chemother 32(11): 1609-13. https://doi.org/10.1128/AAC.32.11.1609

20. DRAGO L, DE VECCHI E, MOMBELLI B, NICOLA L, VALLI M, GISMONDO MR 2001 Activity of levofloxacin and ciprofloxacin against urinary pathogens. J Antimicrob Chemother 48(1): 37-45. https://doi.org/10.1093/jac/48.1.37

21. HOŠTACKA A, ČIŽNAR I 2003 Some properties of Pseudomonas shigelloides with aminoglycosides. Folia Microbiol (Praha) 48(5): 659-63. https://doi.org/10.1007/BF02993475

22. ZHANEL GG, HOBAN DJ, HARDING GK 1992 Subinhibitory antimicrobial concentrations: A review of in vitro and in vivo data. Can J Infect Dis 3(4): 193-201. https://doi.org/10.1155/1992/793607

23. LI X-Z, PLÉSIAT P, NIKAIDO H 2015 The Challenge of EffluxMediated Antibiotic Resistance in Gram-Negative Bacteria. Clin Microbiol Rev 28(2): 337-418.

https://doi.org/10.1128/CMR.00117-14

24. VRANEŠ J 1996 Effect of subinhibitory concentrations of ceftazidime, ciprofloxacin and azithromycin on the hemagglutination and adherence of uropathogenic Escherichia coli strains. Chemother 42(3): 177-85. https://doi.org/10.1159/000239439

25. GORBY GL, MCGEE ZA 1990 Antimicrobial interference with bacterial mechanisms of pathogenicity: Effect of sub-MIC azithromycin on gonococcal pilation and attachment to human epithelial cells. Antimicrob Agents Chemother 34(12): 2445-8.

https://doi.org/10.1128/AAC.34.12.2445

26. FONSECA AP, EXTREMINA C, FONSECA AF, SOUSA JC 2004 Effect of subinhibitory concentration of piperacillin/tazbactam on Pseudomonas aeruginosa. J Med Microbiol 53(Pt 9): 90310. https://doi.org/10.1099/jmm.0.45637-0

27. VIDAYA KC, MALLYA PS, RAO PS 2005 Inhibition of bacterial adhesion by subinhibitory concentrations of antibiotics. Indian J Med Microbiol 23(2): 102-5. https://doi.org/10.4103/0255-0857.16048

28. WOJNICZ D, KLAK M, ADAMSKI R, JANKOWSKI S 2007 Influence of subinhibitory concentrations of amikacin and ciprofloxacin on morphology and adherence ability of uropathogenic strains. Folia Microbiol (Praha) 52(Suppl 4): 429-36. https://doi.org/10.1007/BF02932099 